\title{
The role of viable but non-infectious developmental forms in chlamydial biology
}

\author{
Nicole Borel ${ }^{1 *}$, Andreas Pospischil' ${ }^{1}$, Alan P. Hudson ${ }^{2}$, Jan Rupp ${ }^{3}$ and Robert V. Schoborg ${ }^{4}$ \\ ${ }^{1}$ Department of Pathobiology, Institute of Veterinary Pathology, University of Zurich, Zurich, Switzerland \\ ${ }^{2}$ Department of Immunology and Microbiology, Wayne State University School of Medicine, Michigan, MI, USA \\ ${ }^{3}$ Department of Molecular and Clinical Infectious Diseases, University Hospital Schleswig-Holstein/Campus Lübeck, Lübeck, Germany \\ ${ }^{4}$ Department of Biomedical Sciences, Quillen College of Medicine, East Tennessee State University, Johnson City, TN, USA \\ ${ }^{*}$ Correspondence: n.borel@access.uzh.ch
}

Edited and reviewed by:

Yousef Abu Kwaik, University of Louisville School of Medicine, USA

Keywords: the chlamydial stress response, obligate intracellular, viable but non-infectious, aberrant bodies, chronic disease

Chlamydiae are both intriguing obligate intracellular Gramnegative bacteria and highly successful animal and human pathogens. Their unique biphasic developmental cycle is characterized by the infectious but metabolically less-active elementary body (EB), which initiates infection in a susceptible host cell, and the dividing, intracellular reticulate body (RB). The chlamydiae hijack host cells for their own purposes and, after infection and multiplication, emerge from the host cell and spread their hundreds of progeny EB to infect the next cell. Moreover, chlamydiae are able to survive in an alternative form named the aberrant body $(\mathrm{AB})$ under stressful in vitro conditions. The $\mathrm{AB}$ state has been defined as the hallmark of persistence-which is herein defined as the viable but non-infectious state of the chlamydial developmental cycle. The key questions of whether this $\mathrm{AB}$ form occurs in vivo and if this alternative chlamydial form contributes to clinically significant chronic diseases processes such as inflammation, scarring, and fibrosis are a matter of debate in the chlamydial research community. These controversies led us to assemble a collection of Opinion, Hypothesis and Theory, Perspective, Review, and Primary Research Articles to shed light on recent research findings. This Research Topic showcases the "chlamydial persistent state" from meaning to mechanism on the cellular and human/animal host level.

The critical dissection of the word "persistence" by Bavoil (2014) points out that much more is hidden behind this term than the definition "to stand still permanently" in a susceptible host. For clinicians, this might represent a bacterial infection that: (i) has a subclinical course, (ii) can escape host immune responses; and (iii) is refractory to antibiotic treatment. The lack of specific diagnostic tests for such "invisible" infections might even complicate the definition and detection of these infections. Ongoing asymptomatic infections may even resemble colonization, as suggested by very recent data on chlamydial infections in the gastrointestinal tract. Notably, asymptomatic gastrointestinal chlamydial infections with recurrent chlamydial fecal shedding have been observed in the Veterinary Medical field for decades. For example, subclinical infections with Chlamydia (C.) psittaci in birds are frequent and these "latently" infected birds pose a significant zoonotic risk. Regardless, it is important for investigators in the Chlamydia field to come to agreement regarding the "definition" of what constitutes a persistent chlamydial infection.
The persistence phenotype in vitro may be linked to chronic disease processes in vivo, such as atherosclerosis. C. pneumoniae has been detected in atherosclerotic lesions of human patients by multiple methods and C. pneumoniae-induced disease progression has been demonstrated in rodent models - an association that propelled several antibiotic treatment trials. The opinion article by Campbell and Rosenfeld (2014) summarizes the outcome of these clinical trials and critically reviews their limitations. Unfortunately, the mixed results of these trials and the conclusion that anti-chlamydial antibiotics should not be recommended for treatment of patients with coronary heart disease and peripheral artery disease may have led to the erroneous assumption that $C$. pneumoniae did not play a role in the pathogenesis of atherosclerosis. The advanced nature of atherosclerotic lesions questions not only the effectiveness of antibiotic treatment to eradicate $C$. pneumoniae but also implicates how challenging the diagnosis of the agent may be. Puolakkainen (2013) discusses the difficulty in differentiating between acute and chronic infections due to the lack of reliable "persistence" biomarkers and commercially available tests. Whole proteome assays have been developed for research purposes but need careful validation on selected specimens from well-studied patient populations. The most desirable test to detect persistent infections would be serum-based, an optimistic goal that remains frustratingly out of reach without a better understanding of what persistence actually is and the molecular mechanisms that underly induction, maintenance, and recovery from this non-replicative state (Bavoil, 2014).

Azithromycin and tetracycline/doxycycline are considered the first-line antibiotics to treat genital chlamydial infection. In vitro, it has been shown that penicillin $\mathrm{G}$ exposure induces $C$. trachomatis persistent/aberrant forms. In culture, persistent organisms also resist killing by azithromycin. Because penicillins are commonly utilized to treat other bacterial infections, this may impact patients with concurrent asymptomatic chlamydial infections. Kintner et al. (2014) investigated if commonly prescribed betalactam antibiotics are able to induce $C$. trachomatis serovar E persistence/stress in culture. All penicillins tested induced viable but non-infectious chlamydial forms at clinically relevant concentrations, which might represent one mechanism by which chlamydiae resist antimicrobial therapy in vivo. In a related study, Ong et al. (2013) determined whether the C. trachomatis 
serine protease HtrA (CtHtrA) was required for recovery from penicillin-induced persistence/stress response. CtHtrA is essential for the replicative phase and the addition of a chemical inhibitor (JO146) of CtHtrA was lethal when added to the cultures at mid-replicative cycle. The same inhibitor prevented reversion and recovery from penicillin persistence, demonstrating the essential role of CtHtrA during recovery from stressful conditions. Not only is HtrA the first chlamydial gene shown to play a role in recovery from persistence, HtrA inhibitors might prove to be useful for eradicating both productively replicating and persistent chlamydiae.

Persistent infections have been described in a magnitude of experimental cell culture models_-all of which involve applying some type of stressor to the chlamydial culture. The most well-described culture model of persistence is the IFN- $\gamma$-induced system. In human cells, IFN- $\gamma$ exposure upregulates indoleamine 2,3-dioxygenase (IDO), decreases intracellular tryptophan (Trp), and induces chlamydial persistence. Chlamydial infectivity can be rescued by addition of exogenous Trp. Four manuscripts in this special issue explore novel aspects of IFN-stimulated persistence. Jerchel et al. (2014) explored C. trachomatis persistence and reactivation and host cytokine responses under normoxic and hypoxic conditions. Oxygen concentrations in the female urogenital tract are physiologically low and further diminished by inflammatory processes. This study demonstrated that hypoxia leads to reactivation of INF- $\gamma$-induced persistent $C$. trachomatis infections. Moreover, host immune signaling responses were diminished, as reflected by reduced activation of MAP-kinase p44/42 and reduced expression of pro-inflammatory cytokines IL-6 and IL8. Recent sophisticated comparative evaluation of Chlamydiales proteomes for Trp content is reviewed by Bonner et al. (2014). Selection for higher-than-predicted (Up-Trp) Trp content, compared to lower-than-predicted (Down-Trp) content, is key for the persistent state and phylogenetically different for Chlamydiaceae compared to other chlamydiae families. Previous studies indicate that there is a significant difference in the capacity of ocular and genital C. trachomatis serovars to deal with tryptophan deprivation. Genital serovars encode a functional tryptophan synthase and can use indole to bypass INF- $\gamma$-induced tryptophan starvation. The perturbed vaginal microbiome as a source of indole in vivo is highlighted in the Hypothesis and Theory article by Aiyar et al. (2014). Indole has been found in vaginal secretions from patients with bacterial vaginosis and indole-producing bacteria have been isolated. Thus, when studying genital chlamydial infections in the future, it will be necessary to consider both the elicited host immune response and the local environmental conditions created by the host microbiome. Transferring such knowledge into the patient's situation is reflected in the article by Lewis et al. (2014). These authors were able to show persistent chlamydial growth forms in the human cervix by novel diagnostic approaches. Local IFN- $\gamma$ levels corresponded to chlamydial growth pattern and morphology. This study may pave way to the establishment of potential biomarker panel for assessing the disease outcome in C. trachomatis infected women.

One ultrastructural feature of persistent chlamydiae observed in many cell culture models is production of abundant membrane vesicles (MVs), which are observed both within the inclusion and in the host cell cytoplasm. The potential role of these vesicles in host-pathogen interactions is explored by Frohlich et al. (2014). The generation of MVs is suggested to be an important mechanism for C. trachomatis intracellular survival of stress. MVs are not only present in primary human endocervical epithelial cells infected with $C$. trachomatis, but also in clinical specimens from infected patients (Lewis et al., 2014). The potential function and role of chlamydial MVs in cargo delivery, innate immune response modulation, exchange of genetic material, and intracellular survival under stress conditions calls for more research in this field.

The "chlamydial anomaly" refers to the observation that chlamydial species are sensitive to antibiotics that target the bacterial cell wall, such as penicillin, despite the fact that a functional cell wall has not been detected. This problem is featured in the research article by De Benedetti et al. (2014). The chlamydial genome contains a nearly complete cell wall precursor biosynthesis pathway. Chlamydiaceae genomes encode GlyA which in turn can serve as a source of D-alanine, as exemplified in C. pneumoniae. In view of this finding, future research might help to clarify the structure of cell wall precursor lipid II and the role of chlamydial penicillin-binding proteins in production of penicillin-induced aberrant chlamydial forms.

Finally, host-pathogen interplay between multiple pathogenic microorganisms, such as the porcine epidemic diarrhea virus (PEDV) co-infection model described in the last paper of this collection (Schoborg and Borel, 2014), might more appropriately reflect the in vivo situation than do artificially-induced cell culture models. In particular, this bacterial-viral co-infection has been shown to occur in vivo, can be mimicked in experimental porcine animal models and includes the local environmental conditions of the gut microbiome.

In summary, this collection highlights the recent findings in the field of the "persistent" chlamydial state, which might reflect persistence, re-occurrence, re-infection or colonization in an animal or human host. The collection also illuminates the multifaceted nature of chlamydial persistence and the complexity that results from the existence of different models and multiple factors involved. Moreover, many models have been developed from in vitro data and applicability to the in vivo situation is, as yet, undefined. As outlined by Bavoil (2014), our limited understanding of what persistence is needs further research in the future and will produce greater insight into the host-pathogen interaction.

\section{REFERENCES}

Aiyar, A., Quayle, A. J., Buckner, L. R., Sherchand, S. P., Chang, T. L., Zea, A. H., et al. (2014). Influence of the tryptophan-indole-IFN $\gamma$ axis on human genital Chlamydia trachomatis infection: role of vaginal co-infections. Front. Cell. Infect. Microbiol. 4:72. doi: 10.3389/fcimb.2014.00072

Bavoil, P. M. (2014). What's in a word: the use, misuse, and abuse of the word "persistence" in Chlamydia biology. Front. Cell. Infect. Microbiol. 4:27. doi: 10.3389/fcimb.2014.00027

Bonner, C. A., Byrne, G. I., and Jensen, R. A. (2014). Chlamydia exploit the mammalian tryptophan-depletion defense strategy as a counter-defensive cue to trigger a survival state of persistence. Front. Cell. Infect. Microbiol. 4:17. doi: 10.3389/fcimb.2014.00017

Campbell, L. A., and Rosenfeld, M. E. (2014). Persistent C. pneumoniae infection in atherosclerotic lesions: rethinking the clinical trials. Front. Cell. Infect. Microbiol. 4:34. doi: 10.3389/fcimb.2014.00034 
De Benedetti, S., Bühl, H., Gaballah, A., Klöckner, A., Otten, C., Schneider, T., et al. (2014). Characterization of serine hydroxymethyltransferase GlyA as a potential source of D-alanine in Chlamydia pneumoniae. Front. Cell. Infect. Microbiol. 4:19. doi: 10.3389/fcimb.2014.00019

Frohlich, K. M., Hua, Z., Quayle, A. J., Wang, J., Lewis, M. E., Chou, C.-W., et al. (2014). Membrane vesicle production by Chlamydia trachomatis as an aptive response. Front. Cell. Infect. Microbiol. 4:73. doi: 10.3389/fcimb.2014.00073

Jerchel, S., Kaufhold, I., Schuchardt, L., Shima, K., and Rupp, J. (2014). Host immune responses after hypoxic reactivation of IFN- $\gamma$ induced persistent Chlamydia trachomatis infection. Front. Cell. Infect. Microbiol. 4:43. doi: 10.3389/fcimb.2014.00043

Kintner, J., Lajoie, D., Hall, J., Whittimore, J., and Schoborg, R. V. (2014). Commonly prescribed $\beta$-lactam antibiotics induce $C$. trachomatis persistence/stress in culture at physiologically relevant concentrations. Front. Cell. Infect. Microbiol. 4:44. doi: 10.3389/fcimb.2014.00044

Lewis, M. E., Belland, R. J., AbdelRahman, Y. M., Beatty, W. L., Aiyar, A. A., Zea, A. H., et al. (2014). Morphologic and molecular evaluation of Chlamydia trachomatis growth in human endocervix reveals distinct growth patterns. Front. Cell. Infect. Microbiol. 4:71. doi: 10.3389/fcimb.2014.00071

Ong, V. A., Marsh, J. W., Lawrence, A., Allan, J. A., Timms, P., and Huston, W. M. (2013). The protease inhibitor JO146 demonstrates a critical role for CtHtrA for Chlamydia trachomatis reversion from penicillin persistence. Front. Cell. Infect. Microbiol. 3:100. doi: 10.3389/fcimb.2013.00100
Puolakkainen, M. (2013). Laboratory diagnosis of persistent human chlamydial infection. Front. Cell. Infect. Microbiol. 3:99. doi: 10.3389/fcimb.2013. 00099

Schoborg, R. V., and Borel, N. (2014). Porcine epidemic diarrhea virus (PEDV) co-infection induced chlamydial persistence/stress does not require viral replication. Front. Cell. Infect. Microbiol. 4:20. doi: 10.3389/fcimb.2014.00020

Conflict of Interest Statement: The authors declare that the research was conducted in the absence of any commercial or financial relationships that could be construed as a potential conflict of interest.

Received: 04 July 2014; accepted: 04 July 2014; published online: 24 July 2014.

Citation: Borel N, Pospischil A, Hudson AP, Rupp J and Schoborg RV (2014) The role of viable but non-infectious developmental forms in chlamydial biology. Front. Cell. Infect. Microbiol. 4:97. doi: 10.3389/fcimb.2014.00097

This article was submitted to the journal Frontiers in Cellular and Infection Microbiology.

Copyright (C) 2014 Borel, Pospischil, Hudson, Rupp and Schoborg. This is an openaccess article distributed under the terms of the Creative Commons Attribution License (CC BY). The use, distribution or reproduction in other forums is permitted, provided the original author(s) or licensor are credited and that the original publication in this journal is cited, in accordance with accepted academic practice. No use, distribution or reproduction is permitted which does not comply with these terms. 